\title{
RIGOROUS PASSAGE TO THE LIMIT IN A SYSTEM OF REACTION-DIFFUSION EQUATIONS TOWARDS A SYSTEM INCLUDING CROSS DIFFUSIONS*
}

\author{
FIAMMETTA CONFORTO ${ }^{\dagger}$ AND LAURENT DESVILLETTES $\ddagger$
}

\begin{abstract}
We present in this work a rigorous passage to the limit (in Dimension 1) in a system of three reaction-diffusion equations coming out of population dynamics, towards a system of two reaction-diffusion equations, one of which includes a cross diffusion term.
\end{abstract}

Key words. Cross diffusion, relaxation, SKT model.

AMS subject classifications. 35K57, 35B25, 35Q92, 92D25.

\section{Introduction}

This paper is devoted to the mathematical study of the convergence of a reactiondiffusion system (first introduced by Iida, Mimura, and Ninomiya, cf. [11]) consisting of three equations towards a reaction-cross diffusion system consisting of two equations (introduced by Shigesada, Kawasaki, and Teramoto, cf. [19]), when a relaxation parameter tends to 0 . We are concerned here with systems appearing in problems of population dynamics, with populations evolving through diffusion and a logistic-type reaction kernel (that is, the competition effects are assumed to be represented by quadratic terms).

One of the species appears in two different states, the corresponding populations are described by the number densities $u_{A}:=u_{A}(t, x) \geq 0$ and $u_{B}:=u_{B}(t, x) \geq 0$. An individual can switch from one state to the other with a rate proportional to $1 / \varepsilon$ and depending upon the density (at time $t$ and point $x$ ) of a second species (described by the number density $v:=v(t, x) \geq 0)$.

When they are in the state $B$, the individuals of the first species have a larger diffusion rate than in the state $A$ (for example, $u_{B}$ represents the individuals which are scared or stressed, and they switch from the quieter state $A$ when individuals of the second species compete with them for the resources).

Finally, for the sake of simplicity, we assume that all the individuals are confined in a domain $\Omega$ of $\mathbb{R}^{N}$; that is, we impose homogeneous Neumann boundary conditions.

The following set of equations summarizes the assumptions discussed above:

$$
\begin{array}{r}
\partial_{t} u_{A}^{\varepsilon}-d_{u} \Delta_{x} u_{A}^{\varepsilon}=\left(r_{1}-a_{1}\left(u_{A}^{\varepsilon}+u_{B}^{\varepsilon}\right)-b_{1} v^{\varepsilon}\right) u_{A}^{\varepsilon} \\
+\frac{1}{\varepsilon}\left(k\left(v^{\varepsilon}\right) u_{B}^{\varepsilon}-h\left(v^{\varepsilon}\right) u_{A}^{\varepsilon}\right), \\
\partial_{t} u_{B}^{\varepsilon}-\left(d_{u}+M \alpha\right) \Delta_{x} u_{B}^{\varepsilon}=\left(r_{1}-a_{1}\left(u_{A}^{\varepsilon}+u_{B}^{\varepsilon}\right)-b_{1} v^{\varepsilon}\right) u_{B}^{\varepsilon} \\
-\frac{1}{\varepsilon}\left(k\left(v^{\varepsilon}\right) u_{B}^{\varepsilon}-h\left(v^{\varepsilon}\right) u_{A}^{\varepsilon}\right),
\end{array}
$$

*Received: January 6, 2013; accepted (in revised form): March 23, 2013. Communicated by Shi Jin.

${ }^{\dagger}$ Department of Mathematics and Computer Science, V.le F. Stagno d'Alcontres, 3198166 Messina, Italy (fiammetta.conforto@unime.it).

Work supported by GNFM- INdAM and by research grants of the University of Messina.

${ }^{\ddagger}$ CMLA, ENS Cachan, CNRS, 61 Av. du Pdt. Wilson, 94235 Cachan Cedex, France (desville@ cmla.ens-cachan.fr). 


$$
\begin{array}{r}
\partial_{t} v^{\varepsilon}-d_{v} \Delta_{x} v^{\varepsilon}=\left(r_{2}-a_{2}\left(u_{A}^{\varepsilon}+u_{B}^{\varepsilon}\right)-b_{2} v^{\varepsilon}\right) v^{\varepsilon}, \text { for } t \geq 0, x \in \partial \Omega, \\
\nabla_{x} u_{A}^{\varepsilon}(t, x) \cdot n(x)=0, \quad \nabla_{x} u_{B}^{\varepsilon}(t, x) \cdot n(x)=0, \quad \nabla_{x} v^{\varepsilon}(t, x) \cdot n(x)=0 .
\end{array}
$$

We finally add the initial data (for $x \in \Omega$ )

$$
u_{A}^{\varepsilon}(0, x)=u_{A 0}(x), \quad u_{B}^{\varepsilon}(0, x)=u_{B 0}(x), \quad v^{\varepsilon}(0, x)=v_{0}(x) .
$$

This system was introduced by Iida, Mimura, and Ninomiya in [11] (cf. also [12]) as a prototype of reaction-diffusion systems which, in some limit, lead to a simple reaction-cross diffusion system (here, the cross diffusion appears only in one of the equations of the limiting system).

The strictly positive parameters $r_{1}, a_{1}, b_{1}, r_{2}, b_{2}$, and $a_{2}$ are related to the logistic competition between the individuals of various types, while the strictly positive parameters $d_{u}, d_{u}+M \alpha, d_{v}$ (with $M \alpha>0$ ) are the diffusion rates of the individuals (the reason for considering a constant $M \alpha$ which is a product of two terms will appear later). Finally, $h$ and $k$ are nonnegative functions describing the effect of the presence of individuals of the second species on the possible switch between states $A$ and $B$ of individuals of the first species, while $\varepsilon>0$ is the typical time scale of this switch. In [11] and [12], the assumptions proposed for $h$ and $k$ are the following: $h$ is monotone increasing, $k$ is monotone decreasing, and

$$
\frac{v}{M}=\frac{h(v)}{h(v)+k(v)}
$$

where $M \geq \max \left(\frac{r_{2}}{b_{2}},\|v(0, \cdot)\|_{\infty}\right)$ is an upper bound for the number density of the second species.

We shall in the sequel consider the following somewhat more general assumption.

Assumption 1: The coefficients $r_{1}, r_{2}, a_{1}, b_{2}, b_{1}, a_{2}, d_{u}, d_{v}, \alpha$, and $M$ are strictly positive constants. Moreover, the functions $h, k$ are of class $C^{2}\left(\mathbb{R}_{+} ; \mathbb{R}_{+}\right)$, and satisfy

$$
\{h=0\} \subset\{0\}, \quad\{k=0\} \subset\{0\} .
$$

Note that the typical choices

$$
h(v)=\frac{v}{M}, \quad k(v)=1-\frac{v}{M},
$$

with $M>\max \left(\frac{r_{2}}{b_{2}},\|v(0, \cdot)\|_{\infty}\right)$ satisfy both Assumption 1 and the assumptions of [12]. In the case of $k$, this is obtained thanks to the maximum principle applied to equation (1.3), (cf. Proposition 2.1).

We also introduce the following hypothesis on the initial data.

Assumption 2: We assume that $v_{0} \in H^{1}(\Omega)$,

$$
0<\inf _{\Omega} v_{0} \leq \sup _{\Omega} v_{0}<+\infty
$$

and $u_{A 0} \geq 0, u_{B 0} \geq 0, u_{A 0} \log u_{A 0}, u_{B 0} \log u_{B 0} \in L^{1}(\Omega)$. 
The following proposition states the existence of weak solutions to system (1.1) (1.5), for a given $\varepsilon>0$.

Proposition 1.1. We consider a smooth bounded subset $\Omega \subset \mathbb{R}^{N}$ for $N \in \mathbb{N}^{*}$, a relaxation time $\varepsilon>0$, and coefficients and initial data satisfying assumptions 1 and 2 . Then there exists a weak solution to system (1.1) - (1.5), that is $u_{A}, u_{B} \in L^{2}([0, T] \times \Omega)$ and $v \in L^{\infty}([0, T] \times \Omega)$ for all $T>0$, such that for any $\varphi_{A}, \varphi_{B}, \psi \in C_{c}^{2}\left(\mathbb{R}_{+} \times \bar{\Omega}\right)$ satisfying $\nabla_{x} \varphi_{A} \cdot n=0, \nabla_{x} \varphi_{B} \cdot n=0, \nabla_{x} \psi \cdot n=0$, the following identities hold:

$$
\begin{aligned}
& -\int_{0}^{\infty} \int_{\Omega} u_{A}^{\varepsilon} \partial_{t} \varphi_{A} d x d t-\int_{\Omega} u_{A 0} \varphi_{A}(0, x) d x-d_{u} \int_{0}^{\infty} \int_{\Omega} u_{A}^{\varepsilon} \Delta_{x} \varphi_{A} d x d t \\
= & \int_{0}^{\infty} \int_{\Omega}\left(r_{1}-a_{1}\left(u_{A}^{\varepsilon}+u_{B}^{\varepsilon}\right)-b_{1} v^{\varepsilon}\right) u_{A}^{\varepsilon} \varphi_{A} d x d t \\
& \quad+\frac{1}{\varepsilon} \int_{0}^{\infty} \int_{\Omega}\left(k\left(v^{\varepsilon}\right) u_{B}^{\varepsilon}-h\left(v^{\varepsilon}\right) u_{A}^{\varepsilon}\right) \varphi_{A} d x d t \\
& \quad-\int_{0}^{\infty} \int_{\Omega} u_{B}^{\varepsilon} \partial_{t} \varphi_{B} d x d t-\int_{\Omega} u_{B 0} \varphi_{B}(0, x) d x-\left(d_{u}+M \alpha\right) \int_{0}^{\infty} \int_{\Omega} u_{B}^{\varepsilon} \Delta_{x} \varphi_{B} d x d t \\
= & \int_{0}^{\infty} \quad \int_{\Omega}\left(r_{1}-a_{1}\left(u_{A}^{\varepsilon}+u_{B}^{\varepsilon}\right)-b_{1} v^{\varepsilon}\right) u_{B}^{\varepsilon} \varphi_{B} d x d t \\
& \quad-\frac{1}{\varepsilon} \int_{0}^{\infty} \int_{\Omega}\left(k\left(v^{\varepsilon}\right) u_{B}^{\varepsilon}-h\left(v^{\varepsilon}\right) u_{A}^{\varepsilon}\right) \varphi_{B} d x d t \\
& -\int_{0}^{\infty} \int_{\Omega}^{\varepsilon} v_{t}^{\varepsilon} \psi d x d t-\int_{\Omega} v_{0} \psi(0, x) d x-d_{v} \int_{0}^{\infty} \int_{\Omega} v^{\varepsilon} \Delta_{x} \psi d x d t \\
= & \int_{0}^{\infty} \int_{\Omega}\left(r_{2}-a_{2}\left(u_{A}^{\varepsilon}+u_{B}^{\varepsilon}\right)-b_{2} v^{\varepsilon}\right) v^{\varepsilon} \psi d x d t .
\end{aligned}
$$

This proposition is a consequence of standard theorems of existence for reactiondiffusion equations. We refer for example to [7, 14].

It is believed (cf. [11] and [12]) that when $\varepsilon \rightarrow 0$, the solutions $\left(u_{A}^{\varepsilon}, u_{B}^{\varepsilon}, v^{\varepsilon}\right)$ of system $(1.1)-(1.5)$ converge towards $\left(u_{A}, u_{B}, v\right)$, where

$$
k(v) u_{B}=h(v) u_{A},
$$

and $\left(u_{A}+u_{B}, v\right)$ satisfies the cross diffusion-reaction system (of two equations)

$$
\begin{aligned}
& \partial_{t}\left(u_{A}+u_{B}\right)-\Delta_{x}\left[\left(d_{u}+M \alpha \frac{h(v)}{k(v)+h(v)}\right)\left(u_{A}+u_{B}\right)\right] \\
= & \left(r_{1}-a_{1}\left(u_{A}+u_{B}\right)-b_{1} v\right)\left(u_{A}+u_{B}\right), \\
\partial_{t} v-d_{v} \Delta_{x} v= & \left(r_{2}-a_{2}\left(u_{A}+u_{B}\right)-b_{2} v\right) v .
\end{aligned}
$$

This last system can be seen as a particular case of a class of systems introduced in [19] in order to explain the coexistence of individuals of species which, in the absence of cross diffusion, would satisfy the competitive exclusion principle (cf. [13]). More precisely, with respect to the general case of systems introduced in [19], we are here concerned with the case in which no self diffusion appears, and cross diffusion is present in only one of the equations (that is, from the point of view of the diffusion, the system is triangular).

Our main result is that the convergence of the solutions of the system of three reaction-diffusion equations towards the solutions of the system of two reaction-cross 
diffusion equations indeed holds when the dimension is $N=1$. More precisely, we are able to prove the following theorem.

THEOREM 1.2. We consider a bounded interval $\Omega \subset \mathbb{R}$, and coefficients, initial data satisfying assumptions 1 and 2.

Then a weak solution $\left(u_{A}^{\varepsilon}, u_{B}^{\varepsilon}, v^{\varepsilon}\right)$ to system (1.1) - (1.5) given by Proposition 1.1 (for $\varepsilon>0$ ) converges (up to extraction) when $\varepsilon \rightarrow 0$ towards $\left(u_{A}, u_{B}, v\right)$ for a.e. $(t, x) \in$ $\mathbb{R}_{+} \times \Omega$, and in $L^{2}([0, T] \times \Omega) \times L^{2}([0, T] \times \Omega) \times L^{p}([0, T] \times \Omega)$ strongly for all $p \in[1, \infty[$ and all $T>0$. Moreover, equation (1.13) holds and $\left(u_{A}+u_{B}, v\right)$ is a weak solution of equations (1.14), (1.15) with homogeneous Neumann boundary conditions (for $t \geq 0$, $x \in \partial \Omega)$

$$
\nabla_{x}\left(u_{A}+u_{B}\right)(t, x) \cdot n(x)=0, \quad \nabla_{x} v(t, x) \cdot n(x)=0,
$$

and initial data (for $x \in \Omega$ )

$$
u_{A}(0, x)+u_{B}(0, x)=u_{A 0}(x)+u_{B 0}(x), \quad v(0, x)=v_{0}(x) .
$$

More precisely, for any $\varphi, \psi \in C_{c}^{2}\left(\mathbb{R}_{+} \times \bar{\Omega}\right)$ satisfying $\nabla_{x} \varphi \cdot n=0, \nabla_{x} \psi \cdot n=0$, we have

$$
\begin{aligned}
& -\int_{0}^{\infty} \int_{\Omega}\left(u_{A}+u_{B}\right) \partial_{t} \varphi d x d t-\int_{\Omega}\left(u_{A 0}+u_{B 0}\right) \varphi(0, x) d x \\
& \quad-\int_{0}^{\infty} \int_{\Omega}\left(d_{u}+M \alpha \frac{h(v)}{h(v)+k(v)}\right)\left(u_{A}+u_{B}\right) \Delta_{x} \varphi d x d t \\
= & \int_{0}^{\infty} \int_{\Omega}\left(r_{1}-a_{1}\left(u_{A}+u_{B}\right)-b_{1} v\right)\left(u_{A}+u_{B}\right) \varphi d x d t, \\
& \quad-\int_{0}^{\infty} \int_{\Omega} v \partial_{t} \psi d x d t-\int_{\Omega} v_{0} \psi(0, x) d x-d_{v} \int_{0}^{\infty} \int_{\Omega} v \Delta_{x} \psi d x d t \\
= & \int_{0}^{\infty} \int_{\Omega}\left(r_{2}-a_{2}\left(u_{A}+u_{B}\right)-b_{2} v\right) v \psi d x d t .
\end{aligned}
$$

Finally, the following regularity result holds: $u_{A}, u_{B}$ belong to $L^{2}(\log L)([0, T] \times \Omega)$ for all $T>0$.

Note that this result is known (in any dimension of space) under the extra assumption that $\left(u_{A}^{\varepsilon}, u_{B}^{\varepsilon}, v^{\varepsilon}\right)$ is bounded (uniformly with respect to $\left.\varepsilon\right)$ in $L^{\infty}([0, T] \times \Omega)$ (cf. [11]). To obtain such a property is however still an open problem (at least for global weak solutions).

Note also that the convergence of the steady solutions to (1.1) - (1.4) towards the steady solutions to (1.13) - (1.15) has been thoroughly investigated in [12] from both the numerical and analytical points of view.

Our result is also directly related to a recent and important paper by H. Murakawa (cf. [17]), which deals with much more general systems (in any dimensions) by using energy methods. We briefly explain what are the differences between our result and the theorems proven in [17].

- First, the reaction term in [17] is supposed to be Lipschitz-continuous, whereas ours has a quadratic growth. Note however that the use of the negative sign in $\left(u_{A}^{\varepsilon}+u_{B}^{\varepsilon}\right) u_{A}^{\varepsilon}$ in equation (1.1) [and the same for equation (1.2) and equation (1.3)] would make it possible to use the approach of [17]. 
- Secondly, in the system (9) p. 151 of [17], the reaction-diffusion system which is introduced is somewhat different from (1.1)-(1.3), because the reaction term $f_{i}(\mathbf{u}+\mathbf{v})$ is present only in the equation for $u_{i}$ (and not for $v_{i}$ ) whereas the reaction term appears in both equation (1.1) and equation (1.2) of the present work. It does not seem obvious to extend the estimate (16) p. 154 of [17] to the case when the equation for $v_{i}$ also contains a (quadratically growing) reaction term.

- Thirdly, and maybe most importantly, in [17], the functions $\phi_{i}\left(\right.$ or $\left.\phi_{i j}\right)$ are supposed to have bounded derivatives (Assumption H2 p. 151 of [17]), and this assumption looks unavoidable in the estimates for $\mid\left\langle\nabla z_{2 j}^{\varepsilon}, \nabla \phi_{i j}\left(z^{\varepsilon}\right)\right\rangle$ | and $\left|<\nabla u_{2 j}^{\varepsilon}, \nabla \phi_{i j}\left(z^{\varepsilon}\right)>\right|$ at the end of p. 156. However, in our case, the function $\phi_{2}$ would correspond to an expression growing like $z_{1} z_{2}$, whose derivative with respect to $z_{1}$ is not bounded.

Our result uses techniques involving weak solutions which are typically useful when maximum principles cannot be proven (as it is the case for $u_{A}^{\varepsilon}, u_{B}^{\varepsilon}$ here). Those techniques are based on Lyapounov functionals or duality lemmas for solutions of reaction-diffusion equations (such a lemma was first proven by M. Pierre and D. Schmitt, cf. [16] and [18]). Those techniques are particularly helpful (when dealing with quadratic reaction terms) when they can be used for the equations satisfied by quantities like $u \log u$ instead of $u$. This is the case in [10] or, in the context of the quasi-steady state approximation, in [2] or [3]. They also have been successfully applied in the study of general cross diffusion-reaction equations with small initial data (cf. [1]).

The difficulty in the present paper is that the reaction term is indeed quadratic, but no superlinear Lyapounov functional can easily be exhibited. We shall actually replace it by a functional which does not decrease along the flow of equations (1.1) - (1.4), but whose derivative can nevertheless be uniformly controlled with respect to $\varepsilon$. As a trade-off, this construction will only hold in Dimension 1. We refer to [5] and [6] for the use of entropy-like functionals in the context of equations with cross diffusion and self diffusion.

Let us briefly explain how our result is related to works dealing with the mathematical theory of the limiting system. This system belongs to a general class of equations possibly including cross diffusions and self diffusions, first introduced in [19], and studied by many authors since that time; we refer for example to $[20,6]$ and [4] for recent papers in which many references are presented. In our case, the system has a special triangular structure (as far as cross diffusion is concerned) which leads to a possible partial use of the maximum principle (that is, for $v$ ). Moreover, no self diffusion appears. This case is treated in [15], where the method which is used (presented in Dimension 2) also enables to get existence of (strong, global) solutions in Dimension 1 (with initial data in a slightly smaller space than what is considered in the present work).

We finally provide some remarks about the initial data. Note first that in the limiting process, part of the initial datum is lost, that is, an initial layer will appear if (1.13) is not satisfied initially. Note also that in Assumption 2, the somewhat unnatural condition that $v_{0}$ is bounded from below (by a strictly positive constant) is imposed. This hypothesis seems to be very difficult to eliminate in our proof. Making the weaker hypothesis $\log v_{0} \in L^{1}(\Omega)$, it is possible to show that $v^{\varepsilon}(t, \cdot)$ is bounded from below (by a strictly positive constant) for any $t>0$ (but with bounds which may blow up when $t \rightarrow 0$ ). Consequently, most of our analysis still holds when $t>0$, and one can 
show that (1.14) and (1.15) are still satisfied by the limit of $\left(u_{A}^{\varepsilon}+u_{B}^{\varepsilon}, v^{\varepsilon}\right)$. We were however not able to show that the initial datum (specifically, the one corresponding to $u_{A}+u_{B}$ ) is still satisfied in the limit. Of course, if $v \mapsto h(v)$ and $v \mapsto k(v)$ are both strictly positive, no assumption on the lower bound of $v_{0}$ is required. This case however does not include the most standard choice of $h$ and $k$, that is (1.8).

The rest of the paper is devoted to the proof of Theorem 1.2. We begin in Section 2 with preliminary estimates which hold in any dimension. We turn then in Section 3 to estimates which are valid only in Dimension 1 (Sobolev estimates in Dimension 1 are required there). In Section 4 we present the Lyapounov-like functional which enables us to prove the main estimates of this paper. Then, Section 5 is devoted to the proof of strong compactness results for the family $\left(u_{A}^{\varepsilon}, u_{B}^{\varepsilon}, v^{\varepsilon}\right)_{\varepsilon>0}$. Estimates are finally presented in Section 6 , which prevent this family to concentrate (in $L^{2}$ ). Thanks to the results of the two last sections, strong convergence in $L^{2}$ is obtained for $\left(u_{A}^{\varepsilon}, u_{B}^{\varepsilon}, v^{\varepsilon}\right)_{\varepsilon>0}$, and this is exactly what is needed to pass to the limit in system $(1.1)-(1.5)$.

\section{Proof of Theorem 1.2: Preliminaries}

We begin with a (uniform with respect to $\varepsilon$ ) estimate of $\left(u_{A}^{\varepsilon}, u_{B}^{\varepsilon}, v^{\varepsilon}\right)$, which is valid in all dimensions of space.

Proposition 2.1. We consider a smooth bounded subset $\Omega \subset \mathbb{R}^{N}$ for $N \in \mathbb{N}^{*}$, and coefficients and initial data satisfying assumptions 1 and 2. Then, a weak solution $u_{A}^{\varepsilon}, u_{B}^{\varepsilon}, v^{\varepsilon}$ to system (1.1) - (1.5) given by Proposition 1.1 satisfies, for all $T>0$ (and $i, j \in\{1, . ., N\})$,

$$
\begin{aligned}
& \sup _{\varepsilon>0}\left\|u_{A}^{\varepsilon}\right\|_{L^{2}([0, T] \times \Omega)}<+\infty, \quad \sup _{\varepsilon>0}\left\|u_{B}^{\varepsilon}\right\|_{L^{2}([0, T] \times \Omega)}<+\infty, \\
& \sup _{\varepsilon>0}\left\|v^{\varepsilon}\right\|_{L^{\infty}([0, T] \times \Omega)}<+\infty, \\
& \sup _{\varepsilon>0}\left\|\frac{\partial v^{\varepsilon}}{\partial t}\right\|_{L^{2}([0, T] \times \Omega)}<+\infty, \quad \sup _{\varepsilon>0}\left\|\frac{\partial^{2} v^{\varepsilon}}{\partial x_{i} x_{j}}\right\| \|_{L^{2}([0, T] \times \Omega)}<+\infty, \\
& \sup _{\varepsilon>0} \sup _{t \in[0, T]} \int_{\Omega}\left|\nabla_{x} v^{\varepsilon}(t, x)\right|^{2} d x<+\infty .
\end{aligned}
$$

Proof. Adding (1.1) and (1.2), one gets the equation

$$
\begin{aligned}
& \partial_{t}\left(u_{A}^{\varepsilon}+u_{B}^{\varepsilon}\right)-\Delta_{x}\left(d_{u} u_{A}^{\varepsilon}+\left(d_{u}+M \alpha\right) u_{B}^{\varepsilon}\right) \\
= & \left(r_{1}-a_{1}\left(u_{A}^{\varepsilon}+u_{B}^{\varepsilon}\right)-b_{1} v^{\varepsilon}\right)\left(u_{A}^{\varepsilon}+u_{B}^{\varepsilon}\right) .
\end{aligned}
$$

At this point, it is possible to directly obtain (2.1) by integrating the above equation with respect to $x$, and by using the fact that $a_{1}>0$ (that is, the quadratic term has the right sign).

We also propose an alternative, more complicated proof, which still holds when the reaction term is not quadratic, but which requires an extra assumption on the initial datum. We can indeed write

$$
\partial_{t}\left(u_{A}^{\varepsilon}+u_{B}^{\varepsilon}\right)-\Delta_{x}\left(K^{\varepsilon}\left(u_{A}^{\varepsilon}+u_{B}^{\varepsilon}\right)\right) \leq \frac{r_{1}^{2}}{4 a_{1}}
$$

with

$$
0<d_{u} \leq K^{\varepsilon}:=\frac{d_{u} u_{A}^{\varepsilon}+\left(d_{u}+M \alpha\right) u_{B}^{\varepsilon}}{u_{A}^{\varepsilon}+u_{B}^{\varepsilon}} \leq d_{u}+M \alpha
$$


We can then use the following lemma, due to M. Pierre and D. Schmitt (cf. [16] and $[18])$.

LEMMA 2.2. We consider a smooth bounded subset $\Omega \subset \mathbb{R}^{N}$ for $N \in \mathbb{N}^{*}$, and assume that $\rho:[0, T] \times \Omega \rightarrow[0,+\infty[$ satisfies

$$
\begin{array}{ll}
\partial_{t} \rho-\Delta_{x}(Z \rho) \leq 0 & \text { on } \quad \Omega, \\
\nabla_{x}(\rho Z) \cdot n=0 & \text { on } \quad \partial \Omega,
\end{array}
$$

where $Z:[0, T] \times \Omega \rightarrow \mathbb{R}$ is a function such that $d_{1} \geq Z \geq d_{0}>0$ for some numbers $d_{1}$, $d_{0}$. Then, for all $T>0$,

$$
\|\rho\|_{L^{2}([0, T] \times \Omega)} \leq\left(1+\frac{d_{1}}{d_{0}}\right) T\|\rho(0, \cdot)\|_{L^{2}(\Omega)} .
$$

Lemma 2.2 then yields (2.1), provided that the initial datum for $u_{A}^{\varepsilon}$ and $u_{B}^{\varepsilon}$ lies in $L^{2}$ (and do not depend upon $\varepsilon$ ).

We now turn to the estimates satisfied by $v^{\varepsilon}$. We observe that according to (1.3),

$$
\begin{aligned}
\partial_{t} v^{\varepsilon}-d_{v} \Delta_{x} v^{\varepsilon} & \leq\left(r_{2}-b_{2} v^{\varepsilon}\right) v^{\varepsilon} \\
& \leq \frac{r_{2}^{2}}{4 b_{2}} .
\end{aligned}
$$

Using (1.9) in Assumption 2, we deduce from (2.10) and the maximum principle that estimate (2.2) holds.

Denoting now

$$
\theta^{\varepsilon}:=\left(r_{2}-a_{2}\left(u_{A}^{\varepsilon}+u_{B}^{\varepsilon}\right)-b_{2} v^{\varepsilon}\right) v^{\varepsilon},
$$

we know that thanks to (2.1) and (2.2), for all $T>0$,

$$
\sup _{\varepsilon>0}\left\|\theta^{\varepsilon}\right\|_{L^{2}([0, T] \times \Omega)}<+\infty .
$$

Taking (1.3) into account, we see that (for all $T>0$ )

$$
\sup _{\varepsilon>0}\left\|\frac{\partial v^{\varepsilon}}{\partial t}-d_{v} \Delta_{x} v^{\varepsilon}\right\|_{L^{2}([0, T] \times \Omega)}<+\infty .
$$

Using standard theorems of parabolic regularization (cf. [14] for example), estimate (2.12) implies estimate (2.3).

Multiplying (1.3) by $\Delta_{x} v^{\varepsilon}$, we see that

$$
\Delta_{x} v^{\varepsilon} \partial_{t} v^{\varepsilon}-d_{v}\left(\Delta_{x} v^{\varepsilon}\right)^{2}=\theta^{\varepsilon} \Delta_{x} v^{\varepsilon},
$$

so that (for all $t>0$ )

$$
\begin{aligned}
& \frac{1}{2} \int_{\Omega}\left|\nabla_{x} v^{\varepsilon}(t, x)\right|^{2} d x+d_{v} \int_{0}^{t} \int_{\Omega}\left|\Delta_{x} v^{\varepsilon}(s, x)\right|^{2} d x d s \\
= & -\int_{0}^{t} \int_{\Omega} \Delta_{x} v^{\varepsilon}(s, x) \theta^{\varepsilon}(s, x) d x d s+\frac{1}{2} \int_{\Omega}\left|\nabla_{x} v^{\varepsilon}(0, x)\right|^{2} d x \\
\leq & \frac{1}{2} d_{v} \int_{0}^{t} \int_{\Omega}\left|\Delta_{x} v^{\varepsilon}(s, x)\right|^{2} d x d s+\frac{1}{2 d_{v}} \int_{0}^{t} \int_{\Omega}\left|\theta^{\varepsilon}(s, x)\right|^{2} d x d s+\frac{1}{2} \int_{\Omega}\left|\nabla_{x} v^{\varepsilon}(0, x)\right|^{2} .
\end{aligned}
$$

Using (2.11) and Assumption 2, we get estimate (2.4).

This ends the proof of Proposition 2.1. 


\section{Proof of Theorem 1.2: Dimension-dependent estimates}

We now turn to estimates which do not necessarily hold in higher dimension. We begin with the proof of the following proposition.

Proposition 3.1. We consider a bounded interval $\Omega \subset \mathbb{R}$, and coefficients and initial data satisfying assumptions 1 and 2. Then, a weak solution $\left(u_{A}^{\varepsilon}, u_{B}^{\varepsilon}, v^{\varepsilon}\right)$ to system (1.1) - (1.5) given by Proposition 1.1 satisfies, for all $T>0$,

$$
\sup _{\varepsilon>0}\left\|\partial_{x} v^{\varepsilon}\right\|_{L^{4}([0, T] \times \Omega)}<+\infty .
$$

Proof. This result is a direct consequence of embedding theorems proven in [14], and can in fact be improved (the space $L^{4}$ can be replaced by $L^{6}$ ). We nevertheless propose an elementary proof below.

Using the Sobolev inequality $H^{1}(\Omega) \subset L^{\infty}(\Omega)$, we get the estimate

$$
\sup _{x \in \Omega}\left|\partial_{x} v^{\varepsilon}(t, x)\right|^{2} \leq C(\Omega)\left\{\int_{\Omega}\left|\partial_{x} v^{\varepsilon}(t, x)\right|^{2} d x+\int_{\Omega}\left|\partial_{x x} v^{\varepsilon}(t, x)\right|^{2} d x\right\},
$$

where $C(\Omega)$ is the constant in the Sobolev inequality.

Using estimates (2.3) and (2.4), and integrating with respect to $t \in[0, T]$, we end up with

$$
\sup _{\varepsilon>0} \int_{0}^{T} \sup _{x \in \Omega}\left|\partial_{x} v^{\varepsilon}(t, x)\right|^{2} d t<+\infty .
$$

Interpolating between estimates (2.4) and (3.3), we get

$$
\begin{aligned}
\int_{0}^{T} \int_{\Omega}\left|\partial_{x} v^{\varepsilon}(t, x)\right|^{4} d x d t \leq & \left(\sup _{s \in[0, T]} \int_{\Omega}\left|\partial_{x} v^{\varepsilon}(s, x)\right|^{2} d x\right) \\
& \times\left(\int_{0}^{T} \sup _{y \in \Omega}\left|\partial_{x} v^{\varepsilon}(t, y)\right|^{2} d t\right),
\end{aligned}
$$

which yields estimate (3.1).

This ends the proof of Proposition 3.1.

We now prove bounds from below for $v^{\varepsilon}$.

Proposition 3.2. We consider a bounded interval $\Omega \subset \mathbb{R}$, and coefficients and initial data satisfying assumptions 1 and 2. Then, a weak solution $\left(u_{A}^{\varepsilon}, u_{B}^{\varepsilon}, v^{\varepsilon}\right)$ to system (1.1) - (1.5) given by Proposition 1.1 satisfies, for all $T>0$,

$$
\inf _{\varepsilon>0} \inf _{(t, x) \in[0, T] \times \Omega} h\left(v^{\varepsilon}(t, x)\right)>0, \quad \inf _{\varepsilon>0} \inf _{(t, x) \in[0, T] \times \Omega} k\left(v^{\varepsilon}(t, x)\right)>0 .
$$

Proof. We first observe that (1.3) implies that

$$
\begin{aligned}
\left(\partial_{t}-d_{v} \partial_{x x}\right)\left(\log v^{\varepsilon}\right) & =d_{v} \frac{\left|\partial_{x} v^{\varepsilon}\right|^{2}}{\left(v^{\varepsilon}\right)^{2}}+r_{2}-a_{2}\left(u_{A}^{\varepsilon}+u_{B}^{\varepsilon}\right)-b_{2} v^{\varepsilon} \\
& \geq-a_{2}\left(u_{A}^{\varepsilon}+u_{B}^{\varepsilon}\right)-b_{2} v^{\varepsilon}
\end{aligned}
$$

As a consequence, for any $t \in[0, T], x \in \Omega$,

$$
\log v^{\varepsilon}(t, x) \geq w^{\varepsilon}(t, x),
$$


where $w^{\varepsilon}$ is the solution of

$$
\left(\partial_{t}-d_{v} \partial_{x x}\right) w^{\varepsilon}=-a_{2}\left(u_{A}^{\varepsilon}+u_{B}^{\varepsilon}\right)-b_{2} v^{\varepsilon},
$$

with homogeneous Neumann boundary conditions

$$
\nabla_{x} w^{\varepsilon}(t, x) \cdot n(x)=0,
$$

and the initial condition

$$
w^{\varepsilon}(0, x)=\log v^{\varepsilon}(0, x)
$$

Moreover

$$
\sup _{\varepsilon>0}\left\|\left(\partial_{t}-d_{v} \partial_{x x}\right) w^{\varepsilon}\right\|_{L^{2}([0, T] \times \Omega)}<+\infty
$$

(thanks to estimates $(2.1),(2.2))$ and $\sup _{x \in \Omega}\left[-w^{\varepsilon}(0, x)\right]<+\infty$ (thanks to hypothesis (1.9) in Assumption 2), so that (see for example the appendix in [9])

$$
\inf _{\varepsilon>0} \inf _{(t, x) \in[0, T] \times \Omega} w^{\varepsilon}(t, x)>0,
$$

and finally

$$
\inf _{\varepsilon>0} \inf _{(t, x) \in[0, T] \times \Omega} v^{\varepsilon}(t, x)>0 .
$$

This estimate, together with (1.7) in Assumption 1, yields estimate (3.4).

This ends the proof of Proposition 3.2.

\section{Proof of Theorem 1.2: Entropy estimates}

In this section, we introduce a functional which is close to the entropies used in reaction-diffusion systems coming out of reversible chemistry (cf. for example [8,9]), and which is close to being a Lyapounov functional. Note that it is also reminiscent of functionals used in [6]. As a consequence, we end up with new estimates for the solution to system (1.1) - (1.5). More precisely, we prove the following proposition.

Proposition 4.1. We consider a bounded interval $\Omega \subset \mathbb{R}$, and coefficients and initial data satisfying assumptions 1 and 2. Then, a weak solution $\left(u_{A}^{\varepsilon}, u_{B}^{\varepsilon}, v^{\varepsilon}\right)$ to system (1.1) - (1.5) given by Proposition 1.1 satisfies, for all $T>0$,

$$
\begin{aligned}
& \sup _{\varepsilon>0} \sup _{t \in[0, T]} \int_{\Omega} u_{A}^{\varepsilon}(t, x)\left|\log u_{A}^{\varepsilon}(t, x)\right| d x<+\infty, \\
& \sup _{\varepsilon>0} \sup _{t \in[0, T]} \int_{\Omega} u_{B}^{\varepsilon}(t, x)\left|\log u_{B}^{\varepsilon}(t, x)\right| d x<+\infty, \\
& \sup _{\varepsilon>0} \int_{0}^{T} \int_{\Omega}\left|\partial_{x} \sqrt{u_{A}^{\varepsilon}}\right|^{2} d x d t<+\infty, \\
& \sup _{\varepsilon>0} \int_{0}^{T} \int_{\Omega}\left|\partial_{x} \sqrt{u_{B}^{\varepsilon}}\right|^{2} d x d t<+\infty, \\
& \sup _{\varepsilon>0} \frac{1}{\varepsilon} \int_{0}^{T} \int_{\Omega}\left|\sqrt{k\left(v^{\varepsilon}\right) u_{B}^{\varepsilon}}-\sqrt{h\left(v^{\varepsilon}\right) u_{A}^{\varepsilon}}\right|^{2} d x d t<+\infty .
\end{aligned}
$$


Proof. We introduce the quantity

$$
H^{\varepsilon}=u_{A}^{\varepsilon} \log \left[h\left(v^{\varepsilon}\right)\right]+u_{B}^{\varepsilon} \log \left[k\left(v^{\varepsilon}\right)\right]+u_{A}^{\varepsilon} \log u_{A}^{\varepsilon}+u_{B}^{\varepsilon} \log u_{B}^{\varepsilon}-u_{A}^{\varepsilon}-u_{B}^{\varepsilon} .
$$

The integral of this quantity will be close to a Lyapounov functional. The computation is performed in any dimension. We first observe that

$$
\begin{aligned}
\frac{\partial H^{\varepsilon}}{\partial t}=\frac{h^{\prime}\left(v^{\varepsilon}\right)}{h\left(v^{\varepsilon}\right)} \frac{\partial v^{\varepsilon}}{\partial t} u_{A}^{\varepsilon} & +\frac{k^{\prime}\left(v^{\varepsilon}\right)}{k\left(v^{\varepsilon}\right)} \frac{\partial v^{\varepsilon}}{\partial t} u_{B}^{\varepsilon}+\log \left[h\left(v^{\varepsilon}\right)\right] \frac{\partial u_{A}^{\varepsilon}}{\partial t}+\log \left[k\left(v^{\varepsilon}\right)\right] \frac{\partial u_{B}^{\varepsilon}}{\partial t} \\
& +\log u_{A}^{\varepsilon} \frac{\partial u_{A}^{\varepsilon}}{\partial t}+\log u_{B}^{\varepsilon} \frac{\partial u_{B}^{\varepsilon}}{\partial t} .
\end{aligned}
$$

Then, we compute

$$
\begin{aligned}
& \Delta_{x}\left\{u_{A}^{\varepsilon} \log \left[h\left(v^{\varepsilon}\right)\right]+u_{A}^{\varepsilon} \log u_{A}^{\varepsilon}-u_{A}^{\varepsilon}\right\} \\
& =2 \frac{h^{\prime}\left(v^{\varepsilon}\right)}{h\left(v^{\varepsilon}\right)} \nabla_{x} u_{A}^{\varepsilon} \cdot \nabla_{x} v^{\varepsilon}+\log \left[h\left(v^{\varepsilon}\right)\right] \Delta_{x} u_{A}^{\varepsilon}+\frac{h^{\prime}\left(v^{\varepsilon}\right)}{h\left(v^{\varepsilon}\right)} u_{A}^{\varepsilon} \Delta_{x} v^{\varepsilon} \\
& +\left(\frac{h^{\prime}}{h}\right)^{\prime}\left(v^{\varepsilon}\right) u_{A}^{\varepsilon}\left|\nabla_{x} v^{\varepsilon}\right|^{2}+\log u_{A}^{\varepsilon} \Delta_{x} u_{A}^{\varepsilon}+\frac{\left|\nabla_{x} u_{A}^{\varepsilon}\right|^{2}}{u_{A}^{\varepsilon}},
\end{aligned}
$$

and

$$
\begin{aligned}
& \Delta_{x}\left\{u_{B}^{\varepsilon} \log \left[k\left(v^{\varepsilon}\right)\right]+u_{B}^{\varepsilon} \log u_{B}^{\varepsilon}-u_{B}^{\varepsilon}\right\} \\
&=2 \frac{k^{\prime}\left(v^{\varepsilon}\right)}{k\left(v^{\varepsilon}\right)} \nabla_{x} u_{B}^{\varepsilon} \cdot \nabla_{x} v^{\varepsilon}+\log \left[k\left(v^{\varepsilon}\right)\right] \Delta_{x} u_{B}^{\varepsilon}+\frac{k^{\prime}\left(v^{\varepsilon}\right)}{k\left(v^{\varepsilon}\right)} u_{B}^{\varepsilon} \Delta_{x} v^{\varepsilon} \\
& \quad+\left(\frac{k^{\prime}}{k}\right)^{\prime}\left(v^{\varepsilon}\right) u_{B}^{\varepsilon}\left|\nabla_{x} v^{\varepsilon}\right|^{2}+\log u_{B}^{\varepsilon} \Delta_{x} u_{B}^{\varepsilon}+\frac{\left|\nabla_{x} u_{B}^{\varepsilon}\right|^{2}}{u_{B}^{\varepsilon}} .
\end{aligned}
$$

As a consequence, if we denote

$$
\begin{aligned}
J^{\varepsilon}=d_{u}( & \left.u_{A}^{\varepsilon} \log \left[h\left(v^{\varepsilon}\right)\right]+u_{A}^{\varepsilon} \log u_{A}^{\varepsilon}-u_{A}^{\varepsilon}\right) \\
& +\left(d_{u}+M \alpha\right)\left(u_{B}^{\varepsilon} \log \left[k\left(v^{\varepsilon}\right)\right]+u_{B}^{\varepsilon} \log u_{B}^{\varepsilon}-u_{B}^{\varepsilon}\right),
\end{aligned}
$$

we see that

$$
\begin{aligned}
\frac{\partial H^{\varepsilon}}{\partial t} & -\Delta_{x} J^{\varepsilon} \\
=\log [ & \left.h\left(v^{\varepsilon}\right) u_{A}^{\varepsilon}\right]\left(r_{1}-a_{1}\left(u_{A}^{\varepsilon}+u_{B}^{\varepsilon}\right)-b_{1} v^{\varepsilon}\right) u_{A}^{\varepsilon} \\
& +\log \left[k\left(v^{\varepsilon}\right) u_{B}^{\varepsilon}\right]\left(r_{1}-a_{1}\left(u_{A}^{\varepsilon}+u_{B}^{\varepsilon}\right)-b_{1} v^{\varepsilon}\right) u_{B}^{\varepsilon} \\
& -\frac{1}{\varepsilon}\left[k\left(v^{\varepsilon}\right) u_{B}^{\varepsilon}-h\left(v^{\varepsilon}\right) u_{A}^{\varepsilon}\right]\left(\log \left[k\left(v^{\varepsilon}\right) u_{B}^{\varepsilon}\right]-\log \left[h\left(v^{\varepsilon}\right) u_{A}^{\varepsilon}\right]\right) \\
& +\left\{\frac{h^{\prime}\left(v^{\varepsilon}\right)}{h\left(v^{\varepsilon}\right)} u_{A}^{\varepsilon}+\frac{k^{\prime}\left(v^{\varepsilon}\right)}{k\left(v^{\varepsilon}\right)} u_{B}^{\varepsilon}\right\} \frac{\partial v^{\varepsilon}}{\partial t} \\
& -\left\{d_{u} \frac{h^{\prime}\left(v^{\varepsilon}\right)}{h\left(v^{\varepsilon}\right)} u_{A}^{\varepsilon}+\left(d_{u}+M \alpha\right) \frac{k^{\prime}\left(v^{\varepsilon}\right)}{k\left(v^{\varepsilon}\right)} u_{B}^{\varepsilon}\right\} \Delta_{x} v^{\varepsilon} \\
& -2\left\{d_{u} \frac{h^{\prime}\left(v^{\varepsilon}\right)}{h\left(v^{\varepsilon}\right)} \nabla_{x} u_{A}^{\varepsilon}+\left(d_{u}+M \alpha\right) \frac{k^{\prime}\left(v^{\varepsilon}\right)}{k\left(v^{\varepsilon}\right)} \nabla_{x} u_{B}^{\varepsilon}\right\} \cdot \nabla_{x} v^{\varepsilon} \\
& -\left\{d_{u}\left(\frac{h^{\prime}}{h}\right)^{\prime}\left(v^{\varepsilon}\right) u_{A}^{\varepsilon}+\left(d_{u}+M \alpha\right)\left(\frac{k^{\prime}}{k}\right)^{\prime}\left(v^{\varepsilon}\right) u_{B}^{\varepsilon}\right\}\left|\nabla_{x} v^{\varepsilon}\right|^{2}
\end{aligned}
$$




$$
-d_{u} \frac{\left|\nabla_{x} u_{A}^{\varepsilon}\right|^{2}}{u_{A}^{\varepsilon}}-\left(d_{u}+M \alpha\right) \frac{\left|\nabla_{x} u_{B}^{\varepsilon}\right|^{2}}{u_{B}^{\varepsilon}} .
$$

The estimates are now done in Dimension 1 only.

We observe that, thanks to estimates (2.2), (3.4), and Assumption 1,

$$
\begin{aligned}
|(4.13)| & \leq C \operatorname{st}\left(u_{A}^{\varepsilon}+u_{B}^{\varepsilon}\right)\left|\frac{\partial v^{\varepsilon}}{\partial t}\right|, \\
|(4.14)| & \leq C \operatorname{st}\left(u_{A}^{\varepsilon}+u_{B}^{\varepsilon}\right)\left|\partial_{x x} v^{\varepsilon}\right|, \\
|(4.15)| & \leq C \operatorname{st}\left(\left|\partial_{x} u_{A}^{\varepsilon}\right|+\left|\partial_{x} u_{B}^{\varepsilon}\right|\right)\left|\partial_{x} v^{\varepsilon}\right| \\
& \leq\left[\frac{d_{u}}{2} \frac{\left|\partial_{x} u_{A}^{\varepsilon}\right|^{2}}{u_{A}^{\varepsilon}}+\frac{d_{u}+M \alpha}{2} \frac{\left|\partial_{x} u_{B}^{\varepsilon}\right|^{2}}{u_{B}^{\varepsilon}}\right]+C s t\left|\partial_{x} v^{\varepsilon}\right|^{2}\left(u_{A}^{\varepsilon}+u_{B}^{\varepsilon}\right), \\
|(4.16)| & \leq C \operatorname{st}\left(u_{A}^{\varepsilon}+u_{B}^{\varepsilon}\right)\left|\partial_{x} v^{\varepsilon}\right|^{2},
\end{aligned}
$$

so that

$$
\begin{aligned}
\frac{\partial H^{\varepsilon}}{\partial t}-\Delta_{x} J^{\varepsilon}+\frac{d_{u}}{2} \frac{\left|\partial_{x} u_{A}^{\varepsilon}\right|^{2}}{u_{A}^{\varepsilon}} \quad+\frac{d_{u}+M \alpha}{2} \frac{\left|\partial_{x} u_{B}^{\varepsilon}\right|^{2}}{u_{B}^{\varepsilon}} \\
+\frac{1}{\varepsilon}\left[k\left(v^{\varepsilon}\right) u_{B}^{\varepsilon}-h\left(v^{\varepsilon}\right) u_{A}^{\varepsilon}\right]\left(\log \left[k\left(v^{\varepsilon}\right) u_{B}^{\varepsilon}\right]-\log \left[h\left(v^{\varepsilon}\right) u_{A}^{\varepsilon}\right]\right) \\
\leq \log \left[h\left(v^{\varepsilon}\right) u_{A}^{\varepsilon}\right]\left(r_{1}-a_{1}\left(u_{A}^{\varepsilon}+u_{B}^{\varepsilon}\right)-b_{1} v^{\varepsilon}\right) u_{A}^{\varepsilon} \\
\quad+\log \left[k\left(v^{\varepsilon}\right) u_{B}^{\varepsilon}\right]\left(r_{1}-a_{1}\left(u_{A}^{\varepsilon}+u_{B}^{\varepsilon}\right)-b_{1} v^{\varepsilon}\right) u_{B}^{\varepsilon} \\
+C s t\left(u_{A}^{\varepsilon}+u_{B}^{\varepsilon}\right)\left[\left|\frac{\partial v^{\varepsilon}}{\partial t}\right|+\left|\partial_{x x} v^{\varepsilon}\right|+\left|\partial_{x} v^{\varepsilon}\right|+\left|\partial_{x} v^{\varepsilon}\right|^{2}\right] .
\end{aligned}
$$

Then, we use the elementary inequalities (for $x>0$ )

$$
x \log x-x+1 \geq 0, \quad x \log x-x+1 \leq C \operatorname{st}(1+x|\log x|),
$$

in order to estimate (using (2.2) and (3.4))

$$
\begin{aligned}
& \log \left[h\left(v^{\varepsilon}\right) u_{A}^{\varepsilon}\right]\left(r_{1}-a_{1}\left(u_{A}^{\varepsilon}+u_{B}^{\varepsilon}\right)-b_{1} v^{\varepsilon}\right) u_{A}^{\varepsilon} \\
= & \left\{\left(h\left(v^{\varepsilon}\right) u_{A}^{\varepsilon}\right) \log \left[h\left(v^{\varepsilon}\right) u_{A}^{\varepsilon}\right]-h\left(v^{\varepsilon}\right) u_{A}^{\varepsilon}+1\right\} \cdot \frac{1}{h\left(v^{\varepsilon}\right)}\left(r_{1}-a_{1}\left(u_{A}^{\varepsilon}+u_{B}^{\varepsilon}\right)-b_{1} v^{\varepsilon}\right) \\
& \quad\left(h\left(v^{\varepsilon}\right) u_{A}^{\varepsilon}-1\right) \frac{1}{h\left(v^{\varepsilon}\right)}\left(r_{1}-a_{1}\left(u_{A}^{\varepsilon}+u_{B}^{\varepsilon}\right)-b_{1} v^{\varepsilon}\right) \\
\leq & C s t\left(1+\left(h\left(v^{\varepsilon}\right) u_{A}^{\varepsilon}\right)\left|\log \left[h\left(v^{\varepsilon}\right) u_{A}^{\varepsilon}\right]\right|\right) \frac{r_{1}}{h\left(v^{\varepsilon}\right)}+r_{1} u_{A}^{\varepsilon}+\frac{a_{1}}{h\left(v^{\varepsilon}\right)}\left(u_{A}^{\varepsilon}+u_{B}^{\varepsilon}\right)+\frac{b_{1}}{h\left(v^{\varepsilon}\right)} v^{\varepsilon} \\
\leq & C \operatorname{st}\left(1+\left(u_{A}^{\varepsilon}\right)^{2}+\left(u_{B}^{\varepsilon}\right)^{2}\right) .
\end{aligned}
$$

A similar computation leads to

$$
\log \left[k\left(v^{\varepsilon}\right) u_{B}^{\varepsilon}\right]\left(r_{1}-a_{1}\left(u_{A}^{\varepsilon}+u_{B}^{\varepsilon}\right)-b_{1} v^{\varepsilon}\right) u_{B}^{\varepsilon} \leq C s t\left(1+\left(u_{A}^{\varepsilon}\right)^{2}+\left(u_{B}^{\varepsilon}\right)^{2}\right) .
$$

Integrating (4.22) on $[0, T] \times \Omega$ and using (4.23), (4.24), we end up with

$$
\begin{gathered}
\int_{\Omega} H^{\varepsilon}(T, x) d x+\frac{d_{u}}{2} \int_{0}^{T} \int_{\Omega} \frac{\left|\partial_{x} u_{A}^{\varepsilon}\right|^{2}}{u_{A}^{\varepsilon}} d x d t+\frac{d_{u}+M \alpha}{2} \int_{0}^{T} \int_{\Omega} \frac{\left|\partial_{x} u_{B}^{\varepsilon}\right|^{2}}{u_{B}^{\varepsilon}} d x d t \\
+\frac{1}{\varepsilon} \int_{0}^{T} \int_{\Omega}\left[k\left(v^{\varepsilon}\right) u_{B}^{\varepsilon}-h\left(v^{\varepsilon}\right) u_{A}^{\varepsilon}\right]\left(\log \left[k\left(v^{\varepsilon}\right) u_{B}^{\varepsilon}\right]-\log \left[h\left(v^{\varepsilon}\right) u_{A}^{\varepsilon}\right]\right) d x d t
\end{gathered}
$$




$$
\begin{aligned}
& \leq \int_{\Omega} H^{\varepsilon}(0, x) d x+C s t \int_{0}^{T} \int_{\Omega}\left(1+\left(u_{A}^{\varepsilon}\right)^{2}+\left(u_{B}^{\varepsilon}\right)^{2}\right) d x d t \\
& \quad+C s t \int_{0}^{T} \int_{\Omega}\left(\left|\frac{\partial v^{\varepsilon}}{\partial t}\right|^{2}+\left|\partial_{x} v^{\varepsilon}\right|^{2}+\left|\partial_{x x} v^{\varepsilon}\right|^{2}+\left|\partial_{x} v^{\varepsilon}\right|^{4}\right) d x d t .
\end{aligned}
$$

We now use estimates (2.1), (2.3), (2.4), and (3.1) (and assumptions 1 and 2) in order to get the estimate

$$
\begin{aligned}
& \quad \int_{\Omega} H^{\varepsilon}(T, x) d x+\frac{d_{u}}{2} \int_{0}^{T} \int_{\Omega} \frac{\left|\partial_{x} u_{A}^{\varepsilon}\right|^{2}}{u_{A}^{\varepsilon}} d x d t+\frac{d_{u}+M \alpha}{2} \int_{0}^{T} \int_{\Omega} \frac{\left|\partial_{x} u_{B}^{\varepsilon}\right|^{2}}{u_{B}^{\varepsilon}} d x d t \\
& \quad+\frac{1}{\varepsilon} \int_{0}^{T} \int_{\Omega}\left[k\left(v^{\varepsilon}\right) u_{B}^{\varepsilon}-h\left(v^{\varepsilon}\right) u_{A}^{\varepsilon}\right]\left(\log \left[k\left(v^{\varepsilon}\right) u_{B}^{\varepsilon}\right]-\log \left[h\left(v^{\varepsilon}\right) u_{A}^{\varepsilon}\right]\right) d x d t \\
& \leq \text { Cst. }
\end{aligned}
$$

Using now (2.2) and (3.4), we know that, for some $K>0$,

$$
\left\|\log \left(h\left(v^{\varepsilon}\right)\right)\right\|_{L^{\infty}([0, T] \times \Omega)} \leq K, \quad\left\|\log \left(k\left(v^{\varepsilon}\right)\right)\right\|_{L^{\infty}([0, T] \times \Omega)} \leq K .
$$

So, thanks to the elementary inequality (which holds for all $K>0$ )

$$
\frac{1}{2} y \log y-(K+1) y \geq-\frac{1}{2} e^{2 K+1},
$$

we see that

$$
\begin{aligned}
& \int_{\Omega} H^{\varepsilon}(T, x) d x \\
& \geq \frac{1}{2} \int u_{A}^{\varepsilon} \log u_{A}^{\varepsilon}(T, x) d x+\frac{1}{2} \int u_{B}^{\varepsilon} \log u_{B}^{\varepsilon}(T, x) d x \\
& \quad+\left[\frac{1}{2} \int u_{A}^{\varepsilon} \log u_{A}^{\varepsilon}(T, x) d x+\frac{1}{2} \int u_{B}^{\varepsilon} \log u_{B}^{\varepsilon}(T, x) d x-\int u_{A}^{\varepsilon}(T, x) d x-\int u_{B}^{\varepsilon}(T, x) d x\right. \\
& \left.\quad-\int u_{A}^{\varepsilon}\left|\log h\left(v^{\varepsilon}\right)\right|(T, x) d x-\int u_{B}^{\varepsilon}\left|\log k\left(v^{\varepsilon}\right)\right|(T, x) d x\right] \\
& \geq \frac{1}{2} \int u_{A}^{\varepsilon}\left|\log u_{A}^{\varepsilon}\right|(T, x) d x+\frac{1}{2} \int u_{B}^{\varepsilon}\left|\log u_{B}^{\varepsilon}\right|(T, x) d x \\
& \quad+\int_{u_{A}^{\varepsilon} \leq 1} u_{A}^{\varepsilon} \log u_{A}^{\varepsilon}(T, x) d x+\int_{u_{B}^{\varepsilon} \leq 1} u_{B}^{\varepsilon} \log u_{B}^{\varepsilon}(T, x) d x-\frac{1}{2} e^{2 K+1} \\
& \geq \frac{1}{2} \int u_{A}^{\varepsilon}\left|\log u_{A}^{\varepsilon}\right|(T, x) d x+\frac{1}{2} \int u_{B}^{\varepsilon}\left|\log u_{B}^{\varepsilon}\right|(T, x) d x-2 e^{-1}|\Omega|-\frac{1}{2} e^{2 K+1} .
\end{aligned}
$$

This immediately ensures that estimates (4.1) - (4.4) hold. Finally, estimate (4.5) is a consequence of (4.26) and the elementary inequality (for any $x, y>0$ )

$$
(x-y)(\log x-\log y) \geq \operatorname{Cst}(\sqrt{x}-\sqrt{y})^{2} .
$$

This ends the proof of Proposition 4.1.

\section{Proof of Theorem 1.2: Strong compactness}

We show in this section that any sequence (indexed by $\varepsilon$ ) of solutions to system (1.1) - (1.5) converges a.e. More precisely, we prove the following proposition. 
Proposition 5.1. We consider a bounded interval $\Omega \subset \mathbb{R}$, and coefficients and initial data satisfying assumptions 1 and 2. Then, a weak solution $\left(u_{A}^{\varepsilon}, u_{B}^{\varepsilon}, v^{\varepsilon}\right)$ to system (1.1) - (1.5) given by Proposition 1.1 satisfies (up to extraction) for a.e. $(t, x) \in$ $\mathbb{R}_{+} \times \Omega$,

$$
u_{A}^{\varepsilon}(t, x) \rightarrow u_{A}(t, x), \quad u_{B}^{\varepsilon}(t, x) \rightarrow u_{B}(t, x), \quad v^{\varepsilon}(t, x) \rightarrow v(t, x),
$$

where $v \in L_{\text {loc }}^{\infty}\left(\mathbb{R}_{+} \times \Omega\right), u_{A}, u_{B} \in L_{\text {loc }}^{2}\left(\mathbb{R}_{+} \times \Omega\right)$, and (equivalently to (1.13))

$$
\begin{aligned}
& u_{A}=\frac{k(v)}{k(v)+h(v)}\left(u_{A}+u_{B}\right), \\
& u_{B}=\frac{h(v)}{k(v)+h(v)}\left(u_{A}+u_{B}\right) .
\end{aligned}
$$

Moreover, $\left(u_{A}, u_{B}, v\right)$ satisfies identity (1.17) in the weak formulation.

Proof. According to (2.2) and (2.3), it is clear that $\left(v^{\varepsilon}\right)_{\varepsilon>0}$ belongs to a strongly compact set of $L^{1}([0, T] \times \Omega)$ (for all $T>0$ ). As a consequence, we can extract from $\left(v^{\varepsilon}\right)_{\varepsilon>0}$ a subsequence (still denoted by $\left(v^{\varepsilon}\right)_{\varepsilon>0}$ ) such that

$$
v^{\varepsilon}(t, x) \rightarrow v(t, x) \quad \text { for a.e }(t, x) \in \mathbb{R}_{+} \times \Omega .
$$

Using now (4.3) and (4.4) [and (2.1)], we see that

$$
\sup _{\varepsilon>0} \int_{0}^{T} \int_{\Omega}\left|\partial_{x} u_{A}^{\varepsilon}\right| d x d t<+\infty ; \quad \sup _{\varepsilon>0} \int_{0}^{T} \int_{\Omega}\left|\partial_{x} u_{B}^{\varepsilon}\right| d x d t<+\infty
$$

thanks to the estimate

$$
\int_{0}^{T} \int_{\Omega}\left|\partial_{x} u\right| d x d t \leq\left(\int_{0}^{T} \int_{\Omega} \frac{\left|\partial_{x} u\right|^{2}}{u} d x d t\right)^{1 / 2}\left(\int_{0}^{T} \int_{\Omega}|u| d x d t\right)^{1 / 2} .
$$

According to (2.1), we can extract from the sequences $\left(u_{A}^{\varepsilon}\right)_{\varepsilon>0}$ and $\left(u_{B}^{\varepsilon}\right)_{\varepsilon>0}$ subsequences (still denoted by $\left(u_{A}^{\varepsilon}\right)_{\varepsilon>0}$ and $\left.\left(u_{B}^{\varepsilon}\right)_{\varepsilon>0}\right)$ such that

$$
u_{A}^{\varepsilon} \rightarrow u_{A}, \quad u_{B}^{\varepsilon} \rightarrow u_{B} \quad \text { in } \quad L^{2}([0, T] \times \Omega) \quad \text { weak, for all } T>0,
$$

where $u_{A}$ and $u_{B}$ both belong to $L^{2}([0, T] \times \Omega)$ for all $T>0$.

Thanks to (2.5) (and (2.1), (2.2)), we know that $\frac{\partial}{\partial t}\left(u_{A}^{\varepsilon}+u_{B}^{\varepsilon}\right)$ is bounded in $L^{1}\left([0, T] ; W^{-2,1}(\Omega)\right)$. Interpolating this bound with (5.4) [that is, applying Aubin's lemma], we obtain the strong compactness of $\left(u_{A}^{\varepsilon}+u_{B}^{\varepsilon}\right)_{\varepsilon>0}$ in $L^{1}([0, T] \times \Omega)$ (for all $T>0$ ). Therefore, we can extract from $\left(u_{A}^{\varepsilon}+u_{B}^{\varepsilon}\right)_{\varepsilon>0}$ a subsequence (still denoted by $\left.\left(u_{A}^{\varepsilon}+u_{B}^{\varepsilon}\right)_{\varepsilon>0}\right)$ such that

$$
\left(u_{A}^{\varepsilon}+u_{B}^{\varepsilon}\right)(t, x) \rightarrow\left(u_{A}+u_{B}\right)(t, x) \quad \text { for a.e }(t, x) \in \mathbb{R}_{+} \times \Omega .
$$

At this level, we see that thanks to (2.1), (2.2), (5.1), and (5.7),

$$
\left(u_{A}^{\varepsilon}+u_{B}^{\varepsilon}\right) v^{\varepsilon} \rightarrow\left(u_{A}+u_{B}\right) v,\left(v^{\varepsilon}\right)^{2} \rightarrow v^{2} \text { strongly in } L^{1}([0, T] \times \Omega), \forall T>0 .
$$

We can therefore pass to the limit in (1.12) in order to get identity (1.17). 
But

$$
\begin{aligned}
\int_{0}^{T} \int_{\Omega}\left|k\left(v^{\varepsilon}\right) u_{B}^{\varepsilon}-h\left(v^{\varepsilon}\right) u_{A}^{\varepsilon}\right|^{2} d x d t \leq & \left(\int_{0}^{T} \int_{\Omega}\left|\sqrt{k\left(v^{\varepsilon}\right) u_{B}^{\varepsilon}}-\sqrt{h\left(v^{\varepsilon}\right) u_{A}^{\varepsilon}}\right|^{2} d x d t\right)^{1 / 2} \\
& \times\left(\int_{0}^{T} \int_{\Omega}\left[\sqrt{k\left(v^{\varepsilon}\right) u_{B}^{\varepsilon}}+\sqrt{h\left(v^{\varepsilon}\right) u_{A}^{\varepsilon}}\right]^{2} d x d t\right)^{1 / 2} \\
\leq & \operatorname{Cst\varepsilon }^{1 / 2}
\end{aligned}
$$

thanks to (2.1), (2.2), (4.5), and Assumption 1.

Therefore, up to extraction of a subsequence,

$$
k\left(v^{\varepsilon}(t, x)\right) u_{B}^{\varepsilon}(t, x)-h\left(v^{\varepsilon}(t, x)\right) u_{A}^{\varepsilon}(t, x) \rightarrow 0 \quad \text { for a.e }(t, x) \in \mathbb{R}_{+} \times \Omega .
$$

But

$$
\begin{aligned}
& u_{A}^{\varepsilon}=\frac{k\left(v^{\varepsilon}\right)\left(u_{A}^{\varepsilon}+u_{B}^{\varepsilon}\right)-\left[k\left(v^{\varepsilon}\right) u_{B}^{\varepsilon}-h\left(v^{\varepsilon}\right) u_{A}^{\varepsilon}\right]}{k\left(v^{\varepsilon}\right)+h\left(v^{\varepsilon}\right)}, \\
& u_{B}^{\varepsilon}=\frac{h\left(v^{\varepsilon}\right)\left(u_{A}^{\varepsilon}+u_{B}^{\varepsilon}\right)+\left[k\left(v^{\varepsilon}\right) u_{B}^{\varepsilon}-h\left(v^{\varepsilon}\right) u_{A}^{\varepsilon}\right]}{k\left(v^{\varepsilon}\right)+h\left(v^{\varepsilon}\right)},
\end{aligned}
$$

so that thanks to the convergences (5.7) and (5.10) (and estimate (3.4)), formulas (5.2) and (5.3) hold, together with the convergences in (5.1) for $u_{A}^{\varepsilon}$ and $u_{B}^{\varepsilon}$.

This concludes the proof of Proposition 5.1.

In order to get the weak formulation (1.16), it remains to verify that $\left(u_{A}^{\varepsilon}+u_{B}^{\varepsilon}\right)^{2}$ converges in $L^{1}$ towards $\left(u_{A}+u_{B}\right)^{2}$ (that is, no $L^{1}$ concentration occurs in the sequence $\left.\left(u_{A}^{\varepsilon}+u_{B}^{\varepsilon}\right)^{2}\right)$. In order to do so, we shall provide an estimate of $u_{A}^{\varepsilon}$ and $u_{B}^{\varepsilon}$ in a slightly better space than $L^{2}$.

6. Proof of Theorem 1.2: Non-concentration estimate for $\left(u_{A}^{\varepsilon}+u_{B}^{\varepsilon}\right)^{2}$ and conclusion

We begin with the following proposition.

Proposition 6.1. We consider a bounded interval $\Omega \subset \mathbb{R}$, and coefficients and initial data satisfying assumptions 1 and 2. Then, a weak solution $\left(u_{A}^{\varepsilon}, u_{B}^{\varepsilon}, v^{\varepsilon}\right)$ to system (1.1) - (1.5) given by Proposition 1.1 satisfies, for all $T>0$,

$$
\begin{aligned}
& \sup _{\varepsilon>0} \int_{0}^{T} \int_{\Omega}\left|u_{A}^{\varepsilon}\right|^{2}\left|\log u_{A}^{\varepsilon}\right| d x d t<+\infty, \\
& \sup _{\varepsilon>0} \int_{0}^{T} \int_{\Omega}\left|u_{B}^{\varepsilon}\right|^{2}\left|\log u_{B}^{\varepsilon}\right| d x d t<+\infty .
\end{aligned}
$$

Proof. Using a Sobolev inequality (with constant $C(\Omega)$ ) in Dimension 1, we get (for any $t \geq 0$ )

$$
\begin{aligned}
\sup _{x \in \Omega}\left|u_{A}^{\varepsilon}(t, x)\right| & =\left(\sup _{x \in \Omega}\left|\sqrt{u_{A}^{\varepsilon}(t, x)}\right|\right)^{2} \\
& \leq C s t \int_{\Omega}\left|\partial_{x} \sqrt{u_{A}^{\varepsilon}(t, x)}\right|^{2} d x,
\end{aligned}
$$


so that according to estimate (4.3),

$$
\sup _{\varepsilon>0} \int_{0}^{T}\left(\sup _{x \in \Omega}\left|u_{A}^{\varepsilon}(t, x)\right| d x\right) d t<+\infty
$$

Therefore,

$$
\begin{aligned}
& \sup _{\varepsilon>0} \int_{0}^{T} \int_{\Omega}\left|u_{A}^{\varepsilon}\right|^{2}\left|\log u_{A}^{\varepsilon}\right| d x d t \\
\leq & \sup _{\varepsilon>0} \int_{0}^{T} \sup _{y \in \Omega}\left|u_{A}^{\varepsilon}(t, y)\right|\left(\int_{\Omega} u_{A}^{\varepsilon}(t, x)\left|\log u_{A}^{\varepsilon}(t, x)\right| d x\right) d t \\
\leq & \sup _{\varepsilon>0}\left(\int_{0}^{T} \sup _{y \in \Omega}\left|u_{A}^{\varepsilon}(t, y)\right| d t\right)\left(\sup _{s \in[0, T]} \int_{\Omega}\left|u_{A}^{\varepsilon}(s, x)\right|\left|\log u_{A}^{\varepsilon}(s, x)\right| d x\right) .
\end{aligned}
$$

Using (4.1) and (6.4) [and doing the same proof for $u_{B}^{\varepsilon}$ ] leads to the conclusion of the proof of Proposition 6.1.

We can now provide the conclusion of the proof of Theorem 1.2.

Proof. Thanks to the convergence (5.1) and estimates (2.2), (6.1), and (6.2), we see that $\left(u_{A}^{\varepsilon}, u_{B}^{\varepsilon}, v^{\varepsilon}\right)$ converges (up to extraction) towards $\left(u_{A}, u_{B}, v\right)$ for a.e. $(t, x) \in$ $\mathbb{R}_{+} \times \Omega$, and in $L^{2}([0, T] \times \Omega) \times L^{2}([0, T] \times \Omega) \times L^{p}([0, T] \times \Omega)$ for all $p \in[1, \infty[$ and $T>$ 0 .

Moreover estimates (6.1) and (6.2) ensure that $u_{A}, u_{B}$ belong to $L^{2}(\log L)([0, T] \times \Omega)$ for all $T>0$.

According to Proposition 5.1, the weak formulation (1.17) is satisfied.

Adding (1.10) and (1.11) for $\varphi:=\varphi_{A}=\varphi_{B}$, we end up with the weak formulation

$$
\begin{aligned}
& -\int_{0}^{\infty} \int_{\Omega}\left(u_{A}^{\varepsilon}+u_{B}^{\varepsilon}\right) \partial_{t} \varphi d x d t-\int_{\Omega}\left(u_{A 0}+u_{B 0}\right) \varphi(0, x) d x \\
& -\int_{0}^{\infty} \int_{\Omega}\left(d_{u} u_{A}^{\varepsilon}+\left(d_{u}+M \alpha\right) u_{B}^{\varepsilon}\right) \Delta_{x} \varphi d x d t \\
= & \int_{0}^{\infty} \int_{\Omega}\left(r_{1}-a_{1}\left(u_{A}^{\varepsilon}+u_{B}^{\varepsilon}\right)-b_{1} v^{\varepsilon}\right)\left(u_{A}^{\varepsilon}+u_{B}^{\varepsilon}\right) \varphi d x d t
\end{aligned}
$$

The first term in this identity becomes $-\int_{0}^{\infty} \int_{\Omega}\left(u_{A}+u_{B}\right) \varphi$ in the limit $\varepsilon \rightarrow 0$ while the second term does not depend upon $\varepsilon$.

Using (5.2) and (5.3), we see that $\int_{0}^{\infty} \int_{\Omega}\left(d_{u} u_{A}^{\varepsilon}+\left(d_{u}+M \alpha\right) u_{B}^{\varepsilon}\right) \Delta_{x} \varphi$ converges towards $\int_{0}^{\infty} \int_{\Omega}\left(u_{A}+u_{B}\right)\left(d_{u}+M \alpha \frac{h(v)}{h(v)+k(v)}\right) \Delta_{x} \varphi$.

Recalling (5.8), we see that $\int_{0}^{\infty} \int_{\Omega} v^{\varepsilon}\left(u_{A}^{\varepsilon}+u_{B}^{\varepsilon}\right) \varphi$ converges to $\int_{0}^{\infty} \int_{\Omega} v\left(u_{A}+u_{B}\right) \varphi$.

It is also clear that $\int_{0}^{\infty} \int_{\Omega}\left(u_{A}^{\varepsilon}+u_{B}^{\varepsilon}\right) \varphi$ converges to $\int_{0}^{\infty} \int_{\Omega}\left(u_{A}+u_{B}\right) \varphi$.

Using (6.1) and (6.2), we see that $u_{A}^{\varepsilon}+u_{B}^{\varepsilon}$ converges to $u_{A}+u_{B}$ in $L^{2}([0, T] \times \Omega)$ for all $T>0$, so that $\int_{0}^{\infty} \int_{\Omega}\left(u_{A}^{\varepsilon}+u_{B}^{\varepsilon}\right)^{2} \varphi$ converges to $\int_{0}^{\infty} \int_{\Omega}\left(u_{A}+u_{B}\right)^{2} \varphi$.

All those convergences lead to the weak formulation (1.16), which concludes the proof of Theorem 1.2. 


\section{REFERENCES}

[1] M. Bendahmane, T. Lepoutre, A. Marrocco, and B. Perthame, Conservative cross diffusions and pattern formation through relaxation, J. Math. Pures Appl., 92 (6), 651-667, 2009.

[2] M. Bisi, F. Conforto, and L. Desvillettes, Quasi-steady-state approximation for reactiondiffusion equations, Bull. Inst. Math. Acad. Sin. (N.S.), 2 (4), 823-850, 2007.

[3] D. Bothe and M. Pierre, Quasi-steady-state approximation for a reaction-diffusion system with fast intermediate, J. Math. Anal. Appl., 368 (1), 120-132, 2010.

[4] Y.S. Choi, R. Lui, and Y. Yamada, Existence of global solutions for the Shigesada-KawasakiTermaoto model with strongly coupled cross-diffusion, Disc. Cont. Dyn. Sys., 10 (3), 719-730, 2004.

[5] L. Chen and A. Jüngel, Analysis of a multi-dimensional parabolic population model with strong cross diffusion, SIAM J. Math. Anal., 36, 301-322, 2004.

[6] L. Chen and A. Jüngel, Analysis of a parabolic cross-diffusion population model without self-diffusion, J. Diff. Equ., 224 (1), 39-59, 2006.

[7] L. Desvillettes, About entropy methods for reaction-diffusion equations, Riv. Mat. Univ. Parma, 7 (7), 81-123, 2007.

[8] L. Desvillettes and K. Fellner, Exponential decay toward equilibrium via entropy methods for reaction-diffusion equations, J. Math. Anal. Appl., 319, 157-176, 2006.

[9] L. Desvillettes and K. Fellner, Entropy methods for reaction-diffusion equations: Degenerate diffusion and slowly growing a-priori bounds, Rev. Matem. Iber., 24 (2), 407-432, 2008.

[10] L. Desvillettes, K. Fellner, M. Pierre, and J. Vovelle, Global existence for quadratic systems of reaction-diffusion, Adv. Nonlin. Stud., 7 (3), 491-511, 2007.

[11] M. Iida, M. Mimura, and H. Ninomiya, Diffusion, cross-diffusion and competitive interaction, J. Math. Biol., 53 (4), 617-641, 2006.

[12] H. Izuhara and M. Mimura, Reaction-diffusion system approximation to the cross-diffusion competition system, Hiroshima Math. J., 38 (2), 315-347, 2008.

[13] K. Kishimoto and H.F. Weinberger, The spatial homogeneity of stable equilibria of some reaction-diffusion systems on convex domains, J. Diff. Equ., 58 (1), 15-21, 1985.

[14] O.A. Ladyzhenskaya and N.N. Uraltseva, Linear and quasilinear elliptic equations, Translated from the Russian by Scripta Technica, Inc. Translation editor: Leon Ehrenpreis Academic Press, New York-London, 495, 35-47, 1968.

[15] Y. Lou, W. Ni, and Y. Wu, On the global existence of a cross-diffusion system, Disc. Cont. Dyn. Sys., 4(2), 193-203, 1998.

[16] R.H. Martin and M. Pierre, Nonlinear reaction-diffusion systems, Math. Sci. Engin., 185, 363-398, 1992.

[17] H. Murakawa, A relation between cross-diffusion and reaction-diffusion, Disc. Cont. Dyn. Sys., 5, 147-158, 2012.

[18] M. Pierre and D. Schmitt, Blowup in reaction-diffusion systems with dissipation of mass, SIAM Rev., 42, 93-106, (electronic) 2000.

[19] N. Shigesada, K. Kawasaki, and E. Teramoto, Spatial segregation of interacting species, J. Theo. Biol., 79 (1), 83-99, 1979.

[20] Y. Wang, The global existence of solutions for a cross-diffusion system, Acta Math. Appl. Sin. Engl. Ser., 21(3), 519-528, 2005. 